\title{
Cardiovascular Autonomic Functions and Its Association with Oxidative Stress Marker in Heavy Smoking and Smokeless Tobacco Users of Low Socioeconomic Status
}

\author{
Ravi Babu Katuri', Prabhu N², Gopal Krushna Pal ${ }^{3}$ \\ ${ }^{1}$ PhD Scholar, ${ }^{2}$ Associate Professor, ${ }^{3}$ Senior Professor, Department of Physiology, Jawaharlal Institute of \\ Postgraduate Medical Education \& Research (JIPMER), Puducherry, India
}

\begin{abstract}
Background: Tobacco usage is a major preventable cause for cardiovascular morbidity and mortality. Tobacco is used in both smoking and smokeless forms. Alteration in cardiac autonomic function is a major predisposing factor for cardiovascular risk. There are only limited studies available which assessed autonomic functional status in smokeless tobacco users. This study was aimed to assess and compare the cardiac autonomic activity and oxidative stress in smoking and smokeless tobacco users with controls.

Methods: Sixty seven apparently healthy volunteers from the construction workers of low socioeconomic status were recruited. Those who are having habit of using tobacco in any form $(n=42)$ were categorized into smoking tobacco group $(\mathrm{n}=22)$ based on the smoking index and fagerstrom score for smokeless tobacco group $(n=20)$. Those who never used tobacco in any form were included as control group $(n=25)$. Cardiovascular autonomic function tests were performed and serum malondialdehyde was measured from venous blood sample.

Conclusion: In this study we found that there is definite alteration in cardiac autonomic function which is in correlation with the level of oxidative stress in heavy tobacco users. This alteration is seen more in heavy smokeless tobacco users in compare with smoking tobacco users.
\end{abstract}

Key words: Cardiovascular autonomic functions, smokeless tobacco, malondialdehyde

\section{Introduction}

Tobacco usage is a major preventable cause for morbidity and mortality. The Health Ministry has estimated that $40 \%$ of India's health problems are because of tobacco use and 0.9 million people die per year from the tobacco related diseases in India ${ }^{1}$. Heavy smoking is the commonest cause for ischemic heart diseases which leads to death in the early age of 30-40 years those who are usually to be free from other myocardial risk factors.

\section{Corresponding author:}

\section{Dr. N. Prabhu,}

Associate Professor, Department of Physiology,

Jawaharlal Institute of Postgraduate Medical Education \& Research (JIPMER), Puducherry - 605006.

Phone: 9790980723, E-mail: drprabhu78@yahoo.com
World Health Organization report on global status (2010) says that smoking causes an estimated $71 \%$ of all lung cancer deaths, $42 \%$ of chronic respiratory diseases and nearly $10 \%$ of cardiovascular diseases ${ }^{2}$. Smokeless tobacco causes oral cancer, hypertension, heart diseases and other conditions. Expected deaths due to tobacco use in the year 2030 are around 8 million $^{3}$. Smoking tobacco includes Cigarettes, Beedi, and Cigar etc. Tobacco can be used in the other forms such as smokeless tobacco which includes Khaini, Gutkha, Pan masala, Moist snuff etc. Prevalence of the smokeless tobacco use is $26 \%$ when compared with smoking tobacco is $14 \%$ among adults in India as reported by the Global adult tobacco survey report India ${ }^{4}$. The intensity of tobacco using is associated with the degree of alteration in cardiac autonomic function ${ }^{5}$. However in literature, data on the comparison of autonomic functional status between the 
heavy tobacco smoking and smokeless tobacco users and its association with oxidative stress is lacking. Therefore, this study was designed to assess the impact of heavy tobacco smoking and smokeless tobacco on cardiac autonomic function and its association with oxidative stress among heavy tobacco smoking and smokeless tobacco users.

\section{Materials and Methods}

This was a cross-sectional study conducted in the department of Physiology of tertiary care teaching hospital in India. After obtaining approval of the Post-graduation Research Monitoring Committee and Institute Human Ethics Committee, Sixty seven apparently healthy volunteers from the construction workers between 20-40years were recruited. The sample size was estimated with expected difference of 6 at $5 \%$ level of significance of autonomic function tests and 90\% power using Open Epi software (Version 2.3.1), in which sample size was calculated to be 67 . An informed written consent was obtained from all the participants prior to the commencement of the study. The subjects were chosen with the help of smoking index for the smoking tobacco users and Fagerstrom scoring for smokeless tobacco users. The smoking index was calculated by multiplying the average number of cigarettes which was smoked per day and the duration of the smoking in years ${ }^{6}$. The number of cigarettes meant the average number of cigarettes which was smoked per day in the past seven days. Score $>201$ were included as study group 1. The Fagerström Test for Nicotine Dependence-Smokeless Tobacco (FTND-ST) score was obtained with the questionnaire ${ }^{7}$ of FTND-ST, score $>6$ were included as study group 2. Modified Kuppuswamy socioeconomic status scale 2012 was used to assess the socioeconomic status of all the participants in the study ${ }^{8}$. In this study, we included construction workers of low socioeconomic status. So subjects were divided into three groups;

Control group: Males with age of 20-40 years, who never used tobacco in any form.

Study group1: Males with the age of 20-40 years who are heavy tobacco smokers
Study group 2: Males with the age of 20-40 years who are heavy smokeless tobacco users

(Fagerström Test for Nicotine DependenceSmokeless Tobacco (FTND-ST) score $>6$ )

The subjects were asked to report to the autonomic function testing laboratory of physiology department and were advised to have little breakfast and wear loose fitting clothes. The subjects were told to refrain from medications known to influence cardiovascular system viz anticholinergics, anti-histaminics, over the counter cough and cold medications, diuretics, sympathomimetics and parasympathomimetic agents for 48 hours prior to the study. Subjects were advised to discontinue short acting $\alpha$ and $\beta$ antagonists 24 hours before and long-acting antagonists 48 hours before the test. In case of any adversity in the health of the subjects resulting in poor overnight sleep quality, or any prodromal symptoms of fever and body pain, the test was postponed. The subjects were advised to come after evacuation of bowels and emptying of bladder.

We have recorded height in standing posture with barefoot, using a wall-mounted stadiometer (Easy Care, V.M. Electronics Hardware Ltd.). Weight was measured with a spring balance to the nearest half a kilogram avoiding zero and parallax errors.Body mass index (BMI)was computed by using formula Quetelet's index $=$ Weight $(\mathrm{Kg}) /(\text { Height })^{2}(\mathrm{~m})$.

After 10 min of supine rest, blood pressure (BP) was recorded by oscillometric method using automated BP monitor Omron MX3 (Omron Healthcare Co. Ltd, Kyoto, Japan). This method uses an electronic pressure sensor that reads and evaluates oscillation of the arteries based on the blood flow. Cuff size of Omron automated sphygmomanometer was $121 \mathrm{~mm}$ (width) $\times 446 \mathrm{~mm}$ (length). The cuff was tied about $2 \mathrm{~cm}$ above the cubital fossa and fitted snugly with the arm, approximately at the level of the heart. For each subject, systolic BP (SBP), diastolic BP (DBP) and basal heart rate (BHR) were recorded in the left arm twice with an interval of five minutes between the recordings. For each of these parameters, the average of the two data was considered as the final recording.

(Smoking Index >201) 


\section{Cardiac autonomic function tests - Procedure:}

\section{HR response to standing}

After ten minutes of supine rest (from the basal recording measurements), second recording of lead II Electrocardogram (ECG) was started, and the subjects were asked to stand after 30 seconds with a due marking on the graph. The postural change was obtained within 3 seconds. HR were serially measured for next 5 minutes of stand i.e, immediate, first, second, third, fourth and fifth minute and allowed to rest for $3 \mathrm{~min}$ to achieve baseline HR values. RR tachogram from the ECG recording was retrieved and copied to a Microsoft Excel worksheet. A graphical representation (line diagram) of the RR tachogram was used to identify the minimum and maximum RR interval after standing. Shortest R-R interval at or around the 15 th beat and the longest $\mathrm{R}-\mathrm{R}$ interval at or around the 30th beat was calculated and thereby 30:15 ratio was computed.

\section{HR response to deep breathing}

Deep breathing was performed at the rate of 6 breaths per minute with inspiratory and expiratory cycles for 5 seconds each. During the 3 minutes of rest period after standing, deep breathing synchronized to a voice metronome and if necessary guided by hand movement was demonstrated to the subjects. The subjects performed deep breathing for the next one minute with continuous lead II ECG recording. Subjects were encouraged to perform deep and maximal respiration. A period of 3 min rest was given after the maneuver for the HR and BP to return to basal state. RR tachogram from the corresponding ECG recording was retrieved and transformed graphically into a line diagram on a Microsoft excel worksheet. The maximum and minimum RR interval averaged over 6 cycles of expiration and inspiration was calculated and the ratio was computed as E: I ratio.

\section{$B P$ response to sustained isometric handgrip}

Initially the maximal voluntary contraction (MVC) during sustained isometric handgrip by the subjects was measured using handgrip dynamometer (Inco, Ambala). Then the subjects were instructed regarding sustaining the handgrip at one third of their MVC. The ECG recordings were started and at the fifteenth second subjects were instructed to perform one third of their MVC for 3 minutes. The maximum diastolic blood pressure (DBP) attained during the maneuver was noted. The difference between this highest DBP recorded during sustained handgrip and baseline supine DBP was noted as the pressor response to the test. After the procedure BP and HR were measured after 2 min to confirm if they have returned to basal levels. The magnitude of DBP rise during the maneuver $(\triangle \mathrm{DBP})$ was calculated as the difference between the maximum DBP attained during the handgrip and the supine basal values.

\section{Statistical Analysis}

The data was analyzed by using IBM-SPSS version 20 software. The data is expressed in mean $\pm \mathrm{SD}$. After normality testing, one-way analysis of variance (ANOVA) was performed to compare the parameters between the groups Post doc test was performed by using Bonferroni. The $\mathrm{p}$ value $<0.05$ was considered as significant.

\section{Results}

There was no significant difference in age $(\mathrm{p}=0.560)$, body weight $(\mathrm{p}=0.138)$, and BMI $(\mathrm{p}=0.569)$ between the study groups (Table 1). The basal heart rate was significantly increaed in the smokeless tobacco group $(73.48 \pm 11.87 / \mathrm{min})$ as compared to controls $(66.72 \pm 9.21 / \mathrm{min})$ (Table 1). Among the cardiovascular autonomic function tests, E: I ratio was reduced in both smokeless and smoking tobacco users when compared to controls $(\mathrm{p}=0.039)$. Diastolic blood pressure difference (D DBP) in isometric handgrip test was increased in both smokeless and smoking tobacco users when compared to controls $(\mathrm{p}=0.003)$. Also, 30:15 ratio in heart rate response to standing was increased in both smokeless $(\mathrm{p}=0.498)$ and smoking tobacco users when compared to controls (Table 2). 
Table 1: Comparison of age, anthropometric parameters, heart rate, blood pressure parameters and Socioeconomic status (SES) between controls, smoking tobacco and smokeless tobacco groups.

\begin{tabular}{|c|c|c|c|c|}
\hline Parameter & $\begin{array}{l}\text { Control } \\
\text { Group } \\
(n=25)\end{array}$ & $\begin{array}{l}\text { Smoking Tobacco } \\
\text { Group } \\
(n=22)\end{array}$ & $\begin{array}{l}\text { Smokeless Tobacco } \\
\text { Group } \\
(\mathrm{n}=\mathbf{2 0})\end{array}$ & p value \\
\hline Age & $26.1 \pm 6.11$ & $28.10 \pm 7.13$ & $27.10 \pm 8.15$ & 0.560 \\
\hline Body weight (kg) & $69.25 \pm 10.65$ & $65 \pm 12.09$ & $63.45 \pm 11.93$ & 0.138 \\
\hline BMI (Kg/m2) & $24.13 \pm 3.47$ & $23.21 \pm 3.37$ & $22.98 \pm 3.92$ & 0.569 \\
\hline HR (bpm) & $66.72 \pm 9.21$ & $66.83 \pm 11.53$ & $73.48 \pm 11.87 * \#$ & 0.042 \\
\hline $\mathrm{SBP}(\mathrm{mmHg})$ & $118.15 \pm 10.02$ & $124.61 \pm 16.04$ & $126.32 \pm 13.08$ & 0.131 \\
\hline DBP (mmHg) & $72.3 \pm 8.81$ & $74.79 \pm 10.31$ & $78.5 \pm 11.65$ & 0.170 \\
\hline \multicolumn{5}{|l|}{ SES } \\
\hline Upper (I) & - & - & - & - \\
\hline Upper Middle (II) & - & - & - & - \\
\hline Middle/Lower Middle (III) & - & - & - & - \\
\hline Lower/Upper Lower (IV) & 35 & 45 & 40 & 0.339 \\
\hline Lower (V) & 65 & 55 & 60 & 0.458 \\
\hline
\end{tabular}

Values expressed as mean $\pm \mathrm{SD}$ and percentages (\%); Analysis was done by one-way ANOVA. BMI-Body Mass Index, HR: Heart rate, SBP: Systolic blood pressure, DBP: Diastolic blood pressure; MAP: Mean arterial pressure, RPP: Rate pressure product, SES-Socioeconomic status. The P values less than 0.05 were considered statistically significant.

\#Comparison between controls and smokeless tobacco group, ${ }^{*} \mathrm{P}<0.05$, ${ }^{* *} \mathrm{P}<0.01$

+ Comparison between controls and smoking tobacco group $* \mathrm{P}<0.05, * * \mathrm{P}<0.01$

Table 2: Comparison of cardiovascular autonomic function testing parameters, MDA between controls, smoking tobacco and smokeless tobacco groups

\begin{tabular}{|c|c|c|c|c|}
\hline Parameter & $\begin{array}{l}\text { Control } \\
\text { Group } \\
(n=25)\end{array}$ & $\begin{array}{l}\text { Smoking Tobacco } \\
\text { Group } \\
(\mathrm{n}=22)\end{array}$ & $\begin{array}{l}\text { Smokeless Tobacco } \\
\text { Group } \\
(\mathbf{n}=\mathbf{2 0})\end{array}$ & $p$ value \\
\hline $30: 15$ ratio & $1.38 \pm 0.23$ & $1.45 \pm 0.23$ & $1.55 \pm 0.71$ & 0.498 \\
\hline E:I ratio & $1.74 \pm 0.88$ & $1.39 \pm 0.35$ & $1.22 \pm 0.61 * \#$ & 0.039 \\
\hline D DBP (IHG) & $14.70 \pm 6.47$ & $17.60 \pm 6.26$ & $19.60 \pm 8.76^{* * \#}$ & 0.003 \\
\hline Serum MDA & $2.51 \pm 1.10$ & $2.69 \pm 1.09$ & $3.68 \pm 1.93 * \#$ & 0.026 \\
\hline
\end{tabular}


Values expressed as mean $\pm \mathrm{SD}$; Analysis done by One Way ANOVA. The P values less than 0.05 were considered statistically significant. 30:15 ratio: Ratio between maximum $R R$ interval at $30^{\text {th }}$ beat and minimum RR interval at $15^{\text {th }}$ beat

E:I ratio: ratio of longest $R R$ interval during expiration to the shortest RR interval during inspiration averaged over 6 cycles of respiration, $\triangle \mathrm{DBP}$ : difference in diastolic blood pressure between supine and Isometric hand grip.

+ Comparison between controls and smoking tobacco group, $* * \mathrm{P}<0.01$. \# Comparison between controls and smokeless tobacco group, $* \mathrm{P}<0.05$, ** $\mathrm{P}<0.01$

\section{Discussion}

Since there are no studies till date comparison of cardiovascular autonomic function in smokeless tobacco and smoking tobacco in young adults, our study has been the first in this untrodden path. Tobacco usage in any forms which resulting in cardiovascular diseases like increase in blood pressure, stroke, and myocardial infarctions. In this present study we had used smokeless tobacco tobacco with score more than 6 in the Fagerstrom Nicotine Dependence Score and heavy smoking tobacco users based on the Smoking Index more than 200. The study participants in this study were in the productive life and young at age. There was no significant change in BMI but we noticed a trend in decreasing in BMI in smokeless tobacco group with controls (Table 1). Literatures suggested that nicotine suppresses the hunger feeling which resulting in decrease of the food intake and therefore decrease in body weight ${ }^{9}$. Study participants belong to the construction workers who need a lot of energy to do their work and should have regular intake of food. Irregularities of food intake may affect the quality of life as decreasing in the productivity at work. This present study showed that significant changes were found in the parasympathetic and sympathetic autonomic function tests in the smokeless tobacco and smoking tobacco respectively when compared to control.

In the present study, the basal heart rate found highly significant in the smokeless tobacco group (73.48 $\pm 11.87 / \mathrm{min})$ as compared to controls $(66.72 \pm 9.21 /$ min) (Table 1). The resting heart rate is considered to be a good indicator for overall cardiac health as heart rate is governed by the parasympathetic activity. Literature suggested that increased HR was a marker of the cardiovascular risk and placed a high burden of disability and mortality ${ }^{10}$.

This study reveals that decrease in E:I ratio was observed in the smoking group $(1.39 \pm 0.35)$ and smokeless tobacco group $(1.22 \pm 0.61)$ when compared with controls $(1.74 \pm 0.88)$ as shows decreased vagal reactivity, as E:I ratio represents parasympathetic reactivity.

In Isometric sustained handgrip test, we measured the rise in the DBP, just before the release of the hand grip dynamometer. In the present study, it was noted that the rise in the DBP was significant rise in smoking group $(19.60 \pm 6.26 \mathrm{~mm} \mathrm{Hg})$ and significant reduction in smokeless tobacco group $(11.60 \pm 8.76 \mathrm{~mm}$ of $\mathrm{Hg})$ in comparison with the controls $(14.70 \pm 6.47 \mathrm{~mm}$ of $\mathrm{Hg}$ ). This suggesting increase in sympathetic activity in smoking tobacco group and decrease in sympathetic activity in smokeless tobacco group respectively, reveals that decrease sympathetic reactivity.

It is essential that risk factors associated with heavy and prolong usage of tobacco in any form smoking or smokeless causes the cardiovascular autonomic alterations and sympathovagal imbalance to be detected. To educate and create awareness to public not to use tobacco in any form and inform them the adverse effects and complications of post usage of tobacco, at an early stage to reduce morbidity and mortality. These individuals can be advised to follow to improve quality of life.

In this study, the baseline activities of the parasympathetic and the sympathetic systems were studied, excluding the acute effects of smoking. Study can be extended with large number of samples to establish best results and we did not measure the plasma nicotine, or epinephrine levels and we also did not correlate them with the autonomic changes.

\section{Conclusion}

Our study findings are suggested that there is definite alteration in cardiac autonomic function which is in correlation with the level of oxidative stress in 
heavy tobacco users. In this study we observed that the autonomic changes such as increased sympathetic activation and more vagal withdrawal in both the tobacco users group compared to their apparently healthy counterparts. Also, we might conclude that there is degree of autonomic imbalance and cardiovascular risk is comparatively higher in smokeless tobacco compared to tobacco smokers. Considering public health, tobacco control program should make more vigorous efforts to generate awareness and prevent the incidence of cardiovascular morbidity and mortality in tobacco users.

Conflicts of Interest: The authors declare that there is no conflict of interest and no competing financial interests exist.

Acknowledgments: Authors are thankful to all the study participants who has given consent and participated in the study and we also thank JIPMER for extending financial support for our project.

\section{References}

1. Global Adult Tobacco Survey India report (200910). Ministry of Health and Family Welfare, Government of India, New Delhi. 2010; p-72.

2. Reddy K S, Gupta P C. Reports on Tobacco control in India. Ministry of Health and Family Welfare, Government of India, New Delhi.2010; p-45.

3. Tayade MC, Kulkarni NB. The effect of smoking on the cardiovascular autonomic functions - A cross sectional study. J ClinDiagn Res JCDR; Jul 2013; 7(7):1307-10.

4. Barutcu I, Esen AM, Kaya D, Turkmen M,
Karakaya O, Melek M, et al. Cigarette smoking and heart rate variability: Dynamic influence of parasympathetic and sympathetic maneuvers. Ann Noninvasive Electrocardiol2005;10:324-9.

5. Alyan O, Kacmaz F, Ozdemir O, Maden O, Topaloglu S, Ozbakir C, et al. Effects of cigarette smoking on heart rate variability and plasma N-terminal pro-B-type natriuretic peptide in healthy subjects: Is there the relationship between both markers? Ann Noninvasive Electrocardiol 2008;13:137-44.

6. Bano R, Ahmad N, Mahagaonkar AM. Study of pulmonary functions in smokers and non-smokers in sugarcane harvesters in rural Maharashtra. Walawalkar International Medical Journal. 2014;1(1):33-8.

7. Gupta R, Gurm H, Bartholomew J. Smokeless tobacco and cardiovascular risk. Archives of Internal Medicine, 2004; 164:1845-1849.

8. Kumar BR, Dudala SR, Rao AR. kuppuswamy's Socio-Economic Status Scale-A Revision of Economic Parameter for 2012. pdf. 2014 Apr 01 [cited 2016 Aug 19].

9. Pal GK, Adithan C, Ananthanarayanan PH, Pal P, Nanda N, Thiyagarajan D, et al. Association of sympathovagal imbalance with cardiovascular risks in young prehypertensives. Am J Cardiol2013;112:1757-62.

10. Mohesh MIG, Ratchagan K, Sundaramurthy A. Astudy of short-term heart rate variability in dipping tobacco users. Asian J Med Sci; 2014; 5(3): 91-94. 